EPJ Web of Conferences 59, 03008 (2013)

DOI: $10.1051 /$ epjconf/20135903008

(C) Owned by the authors, published by EDP Sciences, 2013

\title{
Three-dimensional simulations of asymmetric implosion for cone-guided targets
}

\author{
T. Yanagawa ${ }^{1, a}$, H. Sakagami ${ }^{2}$ and H. Nagatomo ${ }^{3}$ \\ ${ }^{1}$ Department of Physics, Nagoya University, Nagoya, Japan \\ ${ }^{2}$ Fundamental Phys. Simulation Research Div., National Institute for Fusion Science, Toki, \\ Japan \\ ${ }^{3}$ Institute of Laser Engineering, Osaka University, Suita, Japan
}

\begin{abstract}
In a fast ignition scheme using a cone-guided target, the fusion target is imploded with the inserted cone. Due to the existence of the cone, asymmetric implosion is not avoidable, which has the possibility to degrade fuel compression efficiency. This paper shows how the fuel compression and temperature at the maximum compression time are affected by changing the initial opening angle and position of the cone by the use of two and three dimensional fluid code.
\end{abstract}

\section{INTRODUCTION}

In fast ignition using a cone-guided target, the target is imploded by high-power lasers, and then the compressed fuel core is ignited by the use of an ultrahigh intense laser. This scheme allows to efficiently heat the fuel core [1], however, the implosion is asymmetric due to the existence of the cone. Therefore, it is required to estimate the effects of the existence of the cone on implosion performance.

To estimate these effects, we perform implosion simulation focused on fluid motion around the cone by assuming the cone as a solid material that is not deformed. The two and three dimensional fluid code IMPACT-2D/3D [2] is used for simulations, which is developed to simulate flows governed by Euler equations and the calculation is carried out on Cartesian grids. Cartesian coordinates do not have a singular point at the origin, therefore, the Cartesian grid is suited to calculate such a complicated flow while crossing the origin. However, the boundary of the cone cannot be adequately treated in the Cartesian grid since the boundary does not conform with the grid line. To overcome this problem, we install the Immersed Boundary (IB) method [3, 4] to IMPACT-2D/3D. The IB method was firstly developed by Peskin to simulate blood flow of cardiac system, and then many associated methods have been researched and developed [5]. The feature of this method is to carry out the entire simulation on Cartesian grids without conforming to the boundary.

The two and three dimensional simulations of the implosion with the cone-guided target are performed by use of IMPACT-2D/3D with the IB method introduced. We measured the density, temperature, and internal energy of the fuel region for estimating the implosion performance. This paper shows the effects of the cone on the implosion performance in the case of changing of the opening angle and the inserted position of the cone.

\footnotetext{
${ }^{a}$ e-mail: yanagawa.takumi@nifs.ac.jp
}

This is an Open Access article distributed under the terms of the Creative Commons Attribution License 2.0, which permits unrestricted use, distribution, and reproduction in any medium, provided the original work is properly cited. 


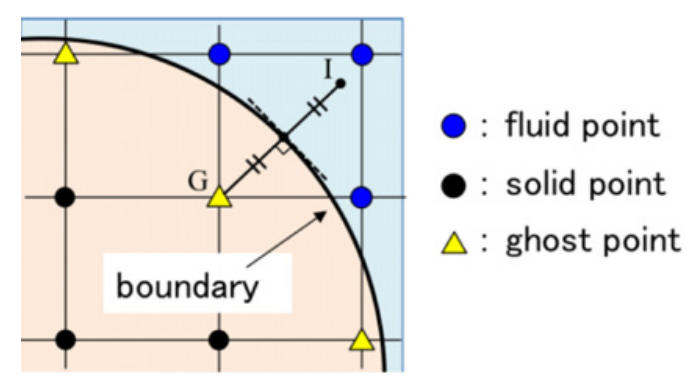

Figure 1. Schematic diagram showing solid, fluid and ghost points in Cartesian grids. $I$ is an image point corresponding to a certain ghost point.

\section{IMMERSED BOUNDARY METHOD}

In the IB method, the boundary of the immersed solid which does not conform with the grid line is treated on Cartesian grids shown in Figure 1. At the pre-processing stage before the calculation of governing equations, all grid points that are located inside the boundary are defined as solid points. Furthermore, the other points, which are located outside the boundary, are defined as fluid points. Any solid points that have at least one fluid point in the neighboring four points are redefined as ghost points. Furthermore, an image point is defined as a mirror projection of the ghost point as shown in Figure 1.

In the main calculation of governing equations with IB method, the value of flow variables $(\phi)$ at each ghost point must be defined for advancing the time step. The value at the ghost point is calculated by using the value at the image point as following. Firstly, the value of $\phi$ at the image point is calculated by an interpolation scheme from the surrounding fluid points. In this paper, the inverse distance weighting method [6] is used as the interpolation scheme. Secondly, the value at the ghost point is evaluated by using the value at the image point to satisfy the boundary condition. For example, given the Dirichlet boundary condition, $\phi=0$, the value at the ghost point is computed by

$$
\phi_{G}=-\phi_{I},
$$

where $\phi_{G}$ and $\phi_{I}$ are the value at the ghost point and the image point, respectively. For the Neumann boundary condition, $\partial \phi / \partial n=0$, where $\partial / \partial n$ is the derivative normal to the boundary, the value at the ghost point is given by

$$
\phi_{G}=\phi_{I} .
$$

The effect of the immersed solid is reflected by setting the ghost point as stated above. Note that the values at all solid points need not to be computed and these points are treated as holes in a computational domain.

\section{SIMULATION}

\subsection{Initial conditions}

The fuel target is assumed to be a shell capsule filled with fuel and the cone is inserted into it as shown in Figure 2(a). This simulation code has no laser absorption calculation. Thus, in order to accelerate the target inward, a high pressure region is needed and introduced to a part of the shell as driven layer. The typical implosion velocity is about $350 \mathrm{~km} / \mathrm{s}$ [7]; we have defined the drive pressure by converting the implosion velocity to kinetic pressure. The initial values are shown in Figure 2(b), where $P_{d}, P_{f}, \rho_{s}$, and $\rho_{f}$ are pressure of the driven layer, pressure of the fuel, density of the shell and density of the fuel, respectively. For two dimensional simulations, we choose $P_{d}=1000 \mathrm{Mbar}$, 

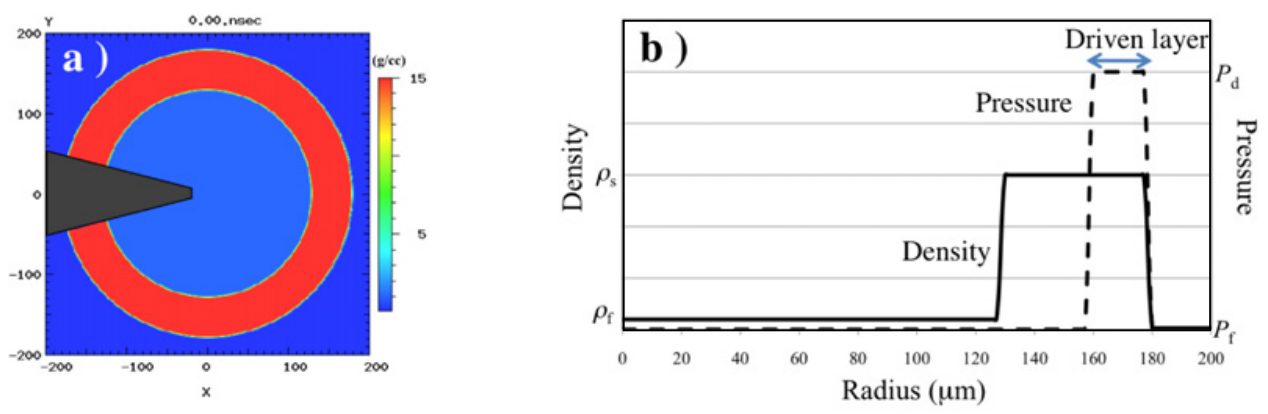

Figure 2. (a) Initial density profile for the two dimensional simulation. (b) Initial conditions of pressure and density. In all regions, velocities are set to zero.
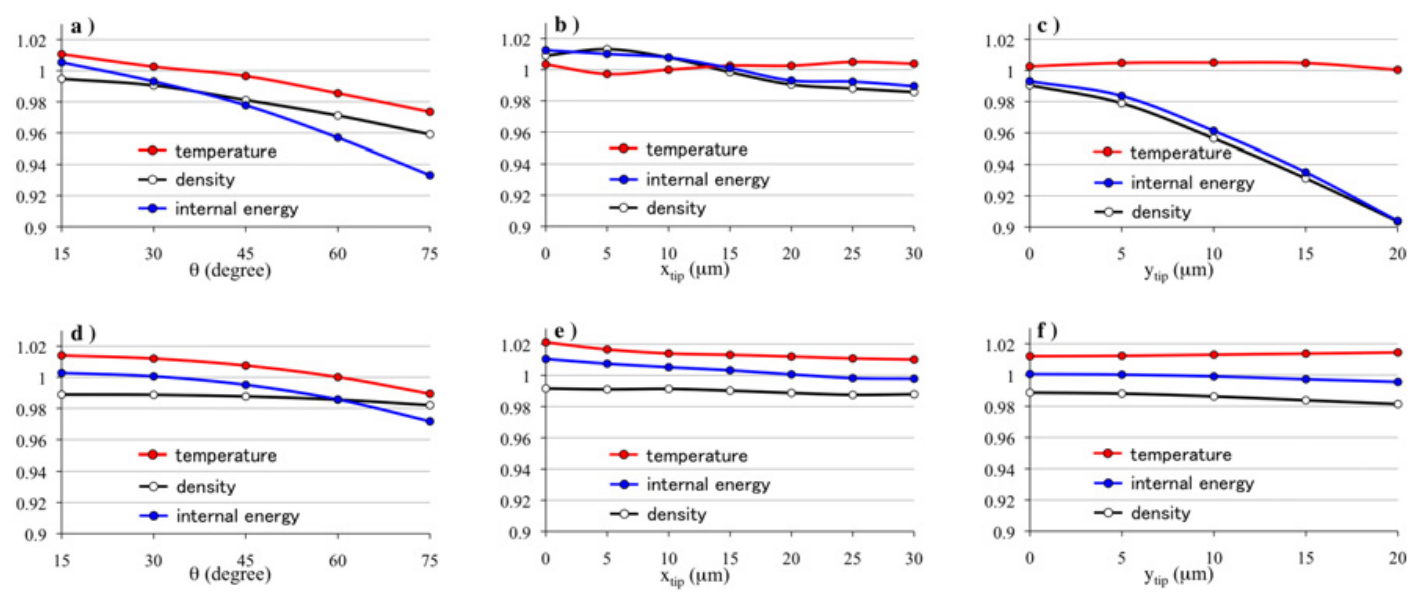

Figure 3. Upper figures are two dimensional results. Lower figures are three dimensional results. The ratios of the average density, temperature and internal energy at the maximum compression compared with the symmetric implosion without the cone are plotted : (a)(d) $\theta$ is variable. $x_{t i p}=20 \mu \mathrm{m}, y_{t i p}=0$; (b)(e) $x_{t i p}$ is variable. $\theta=30^{\circ}$, $y_{t i p}=0$; (c)(f) $y_{t i p}$ is variable. $\theta=30^{\circ}, x_{t i p}=20 \mu \mathrm{m}$.

$P_{f}=1 \mathrm{Mbar}, \rho_{s}=15 \mathrm{~g} / \mathrm{cc}$, and $\rho_{f}=1 \mathrm{~g} / \mathrm{cc}$. The two dimensional simulation corresponds in three dimensional space to implosion of a cylindrical target inserted with a wedge. For three dimensional simulations, we use $P_{d}=8000 \mathrm{Mbar}, P_{f}=1 \mathrm{Mbar}, \rho_{s}=15 \mathrm{~g} / \mathrm{cc}$, and $\rho_{f}=1 \mathrm{~g} / \mathrm{cc}$. The convergence ratio of the fuel radius is about 4 for two dimensional simulations, and about 3 for three dimensional simulations.

\subsection{Results}

Three parameters, $\theta, x_{t i p}$, and $y_{t i p}$ are defined in regard to the cone. $\theta$ is the opening angle of the cone. $x_{\text {tip }}$ is the distance in $x$ direction between the center of the target and the tip of the cone, and $y_{\text {tip }}$ is the distance in $y$ direction between the center and the tip, namely, the offset distance. Note that the tip position is constant in the case of changing the opening angle $\theta$.

To estimate the implosion performance of the cone-guided target, the averaged density and temperature of the fuel region are compared with the case of the symmetric implosion without the cone. The averaged internal energy is also calculated. To determine the fuel region, the interface between the fuel and the shell is defined by a tracking method. Figure 3 shows the averaged density, temperature 

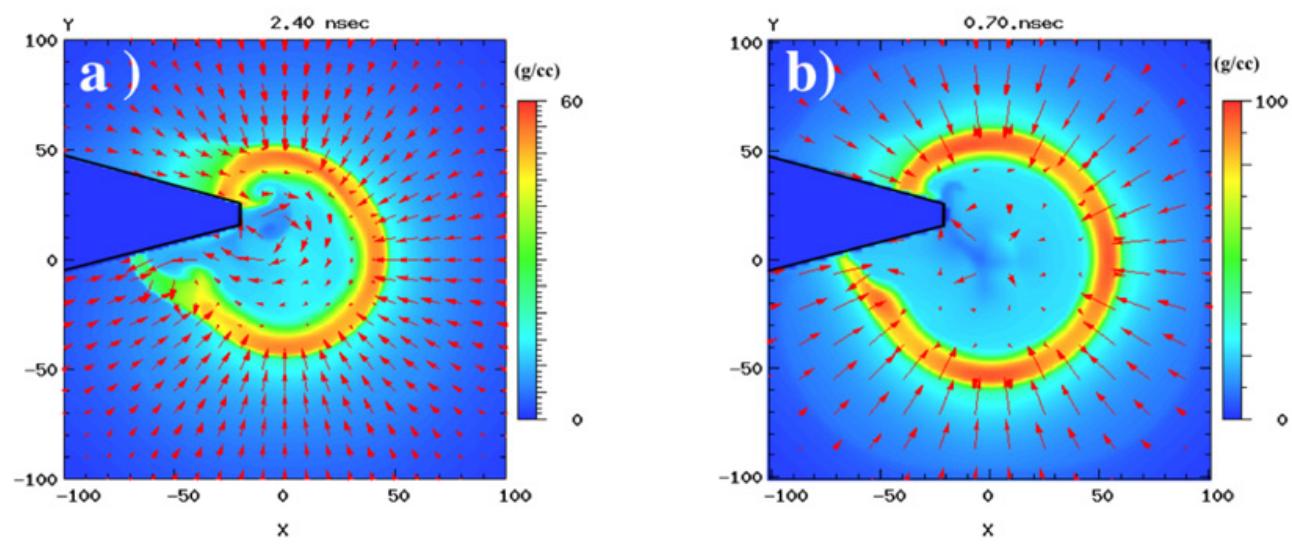

Figure 4. Density contour and flow velocity vectors of the fuel region at the maximum compression when $y_{t i p}=20 \mu \mathrm{m}$ : (a) The two dimensional result. (b) The $x-y$ plane of the three dimensional result.

and internal energy of the fuel region at the maximum compression. These values are normalized by the case of without cone. In the case of two dimensional results (see also the upper graphs of Figure 3), these values tend to decrease as the cone angle $\theta$ increases. As for the change of the offset distance, the temperature does not vary since both the density and internal energy decrease at same rate. This is because the temperature $T$ is proportional to $e_{i n} / \rho$ in ideal gases, where $e_{i n}$ and $\rho$ are the internal energy and the density, respectively.

To investigate the decrease of the density and internal energy in the case of the offset position, we plot the density contour and flow velocity in the fuel region at the maximum compression shown in Figure 4(a). This figure shows the case of $y_{t i p}=20 \mu \mathrm{m}$. It is seen that the shell bursts and the fuel blows out. This results in the decrease of the density. Besides, a vortex structure is observed, which is thought to result from the burst of the shell. The generation of the vortex indicates that the kinetic energy of the fuel region is not efficiently converted to the internal energy. Therefore, the internal energy decreases more than the other cases.

Results of three dimensional simulations show that all fluid parameters are not so affected by the cone compared with two dimensional cases. The most different feature from the two dimensional results is the $y_{t i p}$ dependence. The shell does not burst and vortices do not appear shown in Figure 4(b). In the three dimensional case, stress on the shell is easily dispersed as compared with the two dimensional case because the three dimensional flow is allowed to move in $z$ direction as well as in $x$ and $y$ direction, namely, three dimensional fluid motion has more degrees of freedom than the two dimensional flow. Therefore, the fuel does not break through the shell in this simulation. Additionally, vortices are also hardly generated since the shell does not burst. A more detailed analysis for this problem will be performed in a future study.

\section{References}

[1] R. Kodama et al., Nature 418, 933 (2002)

[2] H. Sakagami, K. Nishihara, Phys. Rev. Lett. 65, 432 (1990)

[3] C.S. Peskin, J. Comput. Phys. 10, 252 (1972)

[4] Y.-h. Tseng, J.H. Ferziger, J. Comput. Phys. 192, 593 (2003)

[5] R. Mittal, G. Iaccarino, Annu. Rev. Fluid Mech. 37, 239 (2005)

[6] R. Franke, Math. Comput. 38, 181 (1982)

[7] S. Atzeni and J. Meyer-ter-Vehn, The Physics of Inertial Fusion (Oxford University Press, New York, 2004) 56 